\title{
Effect of the near-net shape forming on silicon morphology in an Al-Si functionally graded material generated by the centrifugal method
}

\author{
Yasuyoshi Fukui*, Daisaku Nara, Kazuyo Fushimi, Mitsuhiro Nakao, Noriyoshi Kumazawa \\ Graduate School of Science and Engineering, Kagoshima University, Kagoshima, Japan
}

Email address:

fukui@mech.kagoshima-u.ac.jp (Y. Fukui),nara@eng.kagoshima-u.ac.jp (D. Nara), fushimi@eng.kagoshima-u.ac.jp (K. Fushimi), nakao@mech.kagoshima-u.ac.jp (M. Nakao),kumazawa@mech.kagoshima-u.ac.jp (N. Kumazawa)

\section{To cite this article:}

Yasuyoshi Fukui, Daisaku Nara, Kazuyo Fushimi, Mitsuhiro Nakao, Noriyoshi Kumazawa. Effect of the Near-Net Shape Forming on Silicon Morphology in an Al-Si Functionally Graded Material Generated by the Centrifugal Method. International Journal of Materials Science and Applications. Vol. 4, No. 1, 2015, pp. 12-19. doi: 10.11648/j.ijmsa.20150401.13

\begin{abstract}
A work toward practical usage of hypereutectic Al-25 mass\% Si alloy, which exhibits superior properties, as a functionally graded material (FGM) was done. The Al-Si FGM, which is based on the concept of overcoming the limitations imposed by the presence of a hard silicon phase in an aluminum matrix, was generated by a vacuum centrifugal method as a thick-walled tube. Grain coarsening, which is the primary disadvantage of the centrifugal method, was observed. The fraction of silicon phase in the tube unexpectedly varied from greater than 60 mass $\%$ at the outer surface to 15 mass $\%$ at the inner surface because of the greater density of molten silicon compared to that of the eutectic melt. Thus, the outer region of the tube was lighter than the inner region after solidification. FGM billets for near-net shape forming were machined from the thick-walled tube and were formed into an Al-Si FGM cup using a backward extruding. The products of the FGM cup were successfully manufactured in the temperature range from $853 \mathrm{~K}\left(580^{\circ} \mathrm{C}\right)$ to $863 \mathrm{~K}\left(590^{\circ} \mathrm{C}\right)$ through visco-plastic deformation. The fraction of silicon phase in the FGM cup varied from greater than 70 mass $\% \mathrm{Si}$ at the formed cup bottom region to less than $15 \mathrm{mass} \% \mathrm{Si}$ at the cup wall region. Coarse silicon particles were refined irrespective of the pre-existence of elongated spindle-shaped particles under some experimental conditions. The optimum operating conditions were inferred to be high-speed operation at approximately $853 \mathrm{~K}\left(580^{\circ} \mathrm{C}\right)$, which was just above the melting point of the eutectic Al-Si alloy.
\end{abstract}

Keywords: Functionally Graded Material, Hypereutectic Al-Si alloy, Near-Net Shape Forming, Visco-Plastic Flow, Refinement of Particle

\section{Introduction}

The hypereutectic aluminum alloys containing 15 to 25 mass $\%$ Si exhibit excellent wear resistance, low thermal-expansion coefficients, low density, and high strength at elevated temperatures [1]. However, the hard silicon particles distributed in the matrix make these alloys difficult to machine. One of the methods to get the shortage under better control is the implementation of the concept of a functionally graded material (FGM) [2]. FGM are promising materials based on the concept of designing a material to provide a combination of constituents with planar homogeneity and directional inhomogeneity. In the case of a postformation of Al-Si FGM using near-net shape forming, the compositional gradient must overcome the poor workability of the hard and brittle silicon phase through the deformability of the ductile aluminum phase. The Al-Si FGM is expected to exhibit low density combined with superior mechanical properties.

Many methods have been proposed for the manufacturing of FGMs [2], and we have proposed and studied the centrifugal method [3-8]. The product generated by the centrifugal method is a thick-walled tube; the material constituents in the molten metal in the rotating mold experience different centrifugal forces because of their different densities, which results in the generation of a compositional gradient in the radial direction of the tube. The merit of the centrifugal method lies in the possibility of producing large mother FGM at a relatively low cost. In the case of Al-Si alloys, the Al-Si FGM tube exhibits a composition gradient in the thickness direction where the inner region is expected to be rich in silicon because the density of silicon $\left(=2.340 \mathrm{Mg} / \mathrm{m}^{3}\right)$ is lower than that of 
aluminum $\left(=2.700 \mathrm{Mg} / \mathrm{m}^{3}\right)$. The product, for example, can be planed to form a cylinder liner with excellent wear resistance.

The primary disadvantage of the centrifugal method is grain coarsening [4]. The resulting brittle coarse grain makes secondary processing like plastic forming difficult. This shortcoming can be overcome by the application of semi-solid metalworking, which utilizes the semi-solid condition induced during the dissolution process from solid to melt $[9,10]$. Thus, we have been studying the applicability of the concept of using Al-base FGMs and have obtained an interesting results related to the refinement of coarse particle in an Al-base FGM as an additional effect $[7,8]$. Clarification of the optimum operating conditions to produce finer silicon particles is important for the utilization of the superior characteristics of hypereutectic Al-Si alloys because their machinability improves as the silicon particles become finer.

In a series of experiments concerning with FGM, we examined the possibility of producing a near-net shape Al-Si FGM product with fine silicon particles by applying backward extrusion of the Al-Si FGM under a semi-solid condition. The master Al-Si FGM was manufactured by the vacuum centrifugal casting method using hypereutectic Al-25 mass\% $\mathrm{Si}$ alloy. The graded distribution of the silicon phase and capability to induce a fine silicon particle structure for better mechanical properties were studied. We discuss the interaction between the plastic flow resistance and the viscous flow resistance to clarify the optimum forming conditions.

\section{Material and Experimental Procedures}

\subsection{Fabrication of Al-Si FGM}

A master alloy of the Al-Si FGM used in the present study was manufactured by a vacuum centrifugal method using a commercial-purity hypereutectic Al-25 mass\% Si alloy ingot with a chemical composition of 24.7 mass $\%$ Si, 0.14 mass $\%$ $\mathrm{Fe}, 0.00$ mass $\% \mathrm{Cu}$, balance $\mathrm{Al}$. The Al-Si alloy binary phase diagram [11], which is presented in Figure 1, shows that the melting point of the eutectic Al-Si alloy is $850 \mathrm{~K}\left(577^{\circ} \mathrm{C}\right)$. The configuration of the self-made vacuum centrifugal casting system for manufacturing FGM is illustrated in Figure 2. It consists of an alloy melting furnace, an inlet, a mold, etc., which are placed in a chamber with a volume of $0.2 \mathrm{~m}^{3}$. The chamber is designed to be used both under vacuum and under a desired atmosphere to decontaminate the gas dissolved in the alloy.

A batch of alloy lumps with masses less than $1 \mathrm{~kg}$, which were cut from the Al-25 mass\% Si alloy ingot, was melted in the crucible furnace at $1123 \mathrm{~K}\left(850{ }^{\circ} \mathrm{C}\right)$ because the liquidus temperature for the $\mathrm{Al}-25$ mass $\% \mathrm{Si}$ alloy is $1033 \mathrm{~K}\left(760{ }^{\circ} \mathrm{C}\right)$ according to the Al-Si alloy binary phase diagram shown in Figure 1. After the alloy was melted, the lids of the chamber were closed and the inside of the chamber was evacuated using a vacuum pump. The melt was maintained under vacuum for more than $30 \mathrm{~min}$ to remove the gas contained in the alloy melt. The plug, which was set to prevent the melt from leaking from the bottom of the crucible, as shown in Figure 2, was then removed to pour the melt through the inlet into the rotating mold. Both the inlet and mold were pre-heated to approximately $893 \mathrm{~K}\left(620{ }^{\circ} \mathrm{C}\right)$, thereby preventing solidification of the melt before it was completely poured into the mold. Ten minutes later, air was introduced into the chamber and the mold was subsequently subjected to a forced-air cooling.

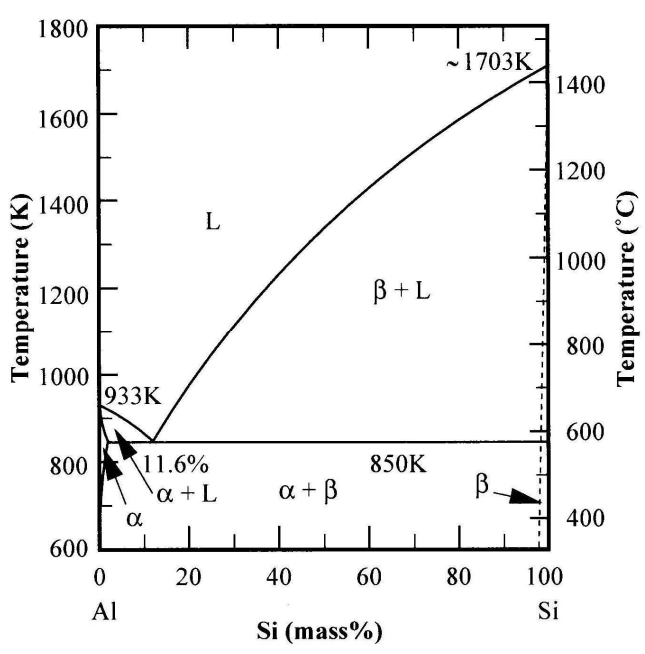

Figure 1. Al-Si alloy binary phase diagram.

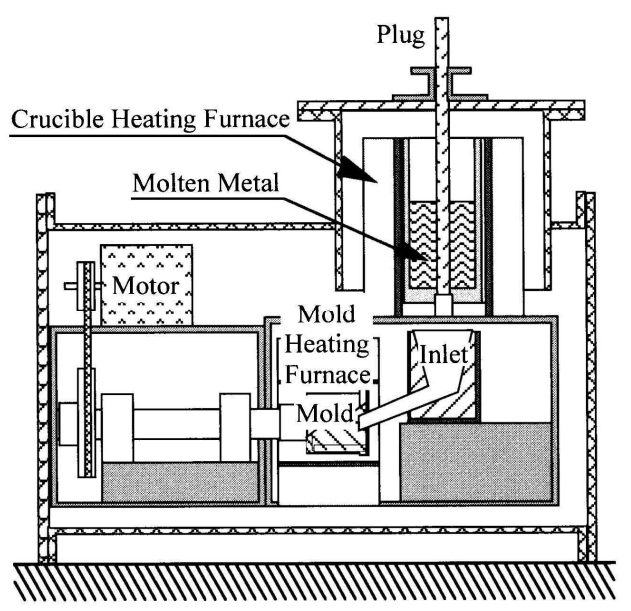

Figure 2. Schematic representation of the FGM manufacturing system in the vacuum chamber.

The effect of centrifugal force on the formation of a composition gradient is evaluated by a $G$ number which is the ratio of the centrifugal force to the force of gravity; the equation is given as follows:

$$
G=2 D N^{2}
$$

where $D$ is the diameter of the casting tube [m] and $N$ is the rate of revolution $\left[\mathrm{s}^{-1}\right]$. In this study $D=9 \times 10^{-2} \mathrm{~m}$ and $N=$ $20 \mathrm{~s}^{-1}$ (i.e., $1200 \mathrm{rpm}$ ); the molten alloy experienced 72 times the force of gravity for forming a composition gradient in the tube radial direction. To prevent the oxidization, the molten alloy was solidified in the vacuum and finally allowed to cool down in the atmosphere. A thick-walled tube with $90 \mathrm{~mm}$ 
diameter $\times 90 \mathrm{~mm}$ length $\times 13 \mathrm{~mm}$ thickness was produced through a finish machining. From the molten alloy, silicon was initially nucleated; the Al-Si FGM structure was obtained as predicted by the $\mathrm{Al}-\mathrm{Si}$ alloy binary phase diagram.

\subsection{Process of Semi-Solid Forming}

A thick-walled Al-Si FGM tube was cut into four bars with a circular arc cross-section, as illustrated in Figure 3. The bar was then subjected to hot working, and a partially flat plate was obtained for the preparation of FGM billets for the near-net shape forming. From the FGM plate, billets of $40 \mathrm{~mm}$ diameter $\times 12 \mathrm{~mm}$ thickness were cut using an electro-discharge machine such that the thickness direction coincided with the radial direction of the original FGM tube. The billet was kept in a container such that a silicon-rich layer formed at the outer plane of the FGM cup after the backward extruding as illustrated in Figure 4 [7], and was then subjected to backward extruding using both a $200-\mathrm{kN}$ hydraulic press and a $100-\mathrm{kN}$ screw-driven universal high-temperature tension-compression machine (SHIMADZU AG-100kNX ${ }^{\mathrm{TM}}$ ). An FGM cup with an outer diameter of $40 \mathrm{~mm}$, an inner diameter $35 \mathrm{~mm}$, a bottom thickness of $3 \mathrm{~mm}$, and a height of $40 \mathrm{~mm}$ was then extruded. The FGM cups always stuck firmly between the container and the punch because of a difference between the thermal expansion coefficients of the hot work tool steel and the Al-25 mass\% Si alloy. Thus, both the punch and the container were produced in a slightly tapered shape. Furthermore, to facilitate removal of the cup from the punch, we utilized a thermal misfit to create a gap at the interface between the FGM cup and the steel punch.

In a typical preparation using the hydraulic press, the container with both billet and punch was heated and maintained at the test temperature for $30 \mathrm{~min}$ in a crucible furnace. The container was then placed on a press machine, and the billet was extruded into an FGM cup in less than $1 \mathrm{~s}$ for a compression of $9 \mathrm{~mm}$. The test temperatures were selected $848 \mathrm{~K}\left(575^{\circ} \mathrm{C}\right), 853 \mathrm{~K}\left(580^{\circ} \mathrm{C}\right), 863 \mathrm{~K}\left(590^{\circ} \mathrm{C}\right)$, and $873 \mathrm{~K}\left(600{ }^{\circ} \mathrm{C}\right)$ and the temperature was monitored via the output of a thermocouple inserted into the bottom of the container; the temperature measurement error was within \pm 2 $\mathrm{K}$. In addition to an experiment using the hydraulic press, a screw-driven universal tension-compression machine was used for constant compression rate tests to evaluate the effect of the working speed precisely on the particle structure of the formed silicon. The test conditions were combinations of crosshead speed of 1,100 , and $500 \mathrm{~mm} / \mathrm{min}$ and temperatures of $853 \mathrm{~K}\left(580{ }^{\circ} \mathrm{C}\right)$ and $863 \mathrm{~K}\left(590^{\circ} \mathrm{C}\right)$ referring to the results of the hydraulic press. The die and billet set for the backward extruding were heated in an electric furnace for $30 \mathrm{~min}$ and the compression load was subsequently applied at a given index temperature. The time required to compress 9 $\mathrm{mm}$ was $9 \mathrm{~min}, 5.4 \mathrm{~s}$, and $1.08 \mathrm{~s}$ for crosshead speeds of 1 , 100 , and $500 \mathrm{~mm} / \mathrm{min}$, respectively. The microstructure of both the FGM cup (after semi-solid forming) and the billet (before semi-solid forming) were observed using an optical microscope.

\section{Results and Discussion}

\subsection{Microstructure and Composition Gradient of the Al-Si FGM}

The morphology and distribution of the silicon phase in the Al-Si FGM investigated in this study were examined using a conventional optical microscope; typical micrographs are shown in Figure 5. The left side of the zone in Figure 5 corresponds to the outer surface region of the FGM tube. In Figure 5, the white parts are the $\alpha$-aluminum rich phase, i.e., the eutectic Al-Si phase, and the dark particles are the $\beta$-silicon phase, which was observed both within $6 \mathrm{~mm}$ from the outer surface and within $1 \mathrm{~mm}$ from inner surface of the tube. The average length of the spindle-shaped silicon particles at the tube outer zone was $1.25 \mathrm{~mm}$. Grain coarsening of silicon phase which is the primary disadvantage of the centrifugal method [4] was confirmed.

The variation of the fraction of the silicon phase, $V_{f}$, at each position was calculated using the rule of mixture under the assumption that the structure is the $(\alpha+\beta)$ phase, as shown in Figure 1. The equation is given as follows:

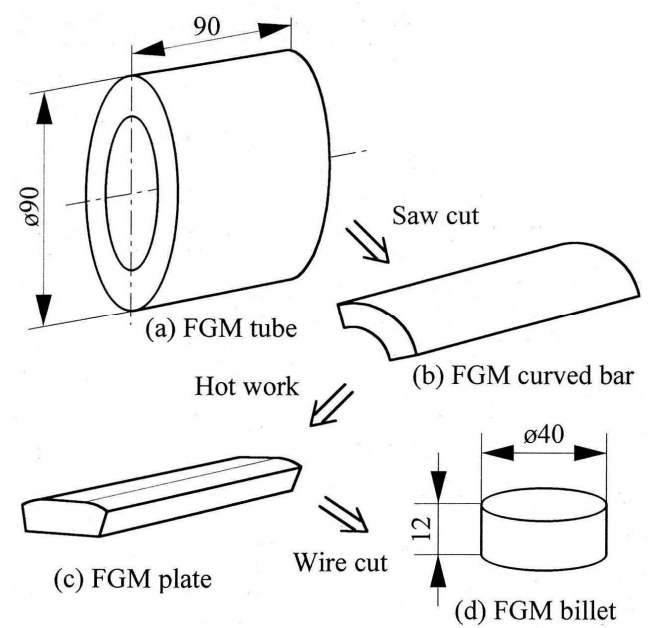

Figure 3. Schematic representation used to prepare the FGM billet preparation from Al-Si FGM thick-walled tube.

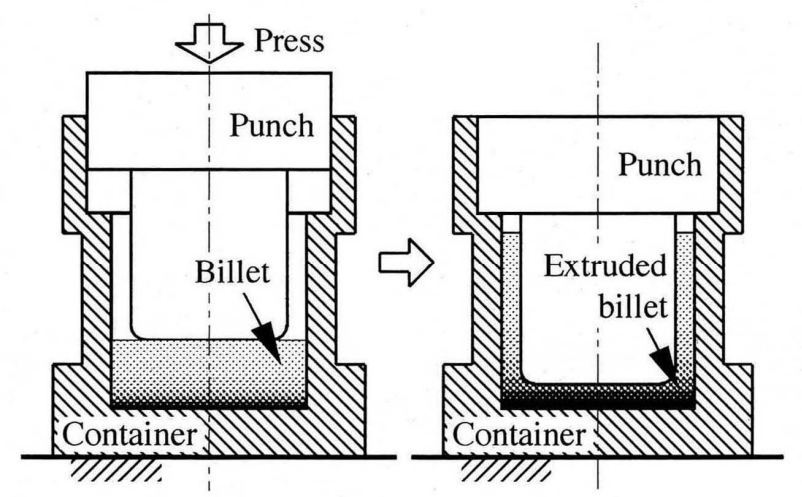

(a) Before forming

(b) After forming

Figure 4. Schematic representation of the backward extruding process for Al-Si FGM 


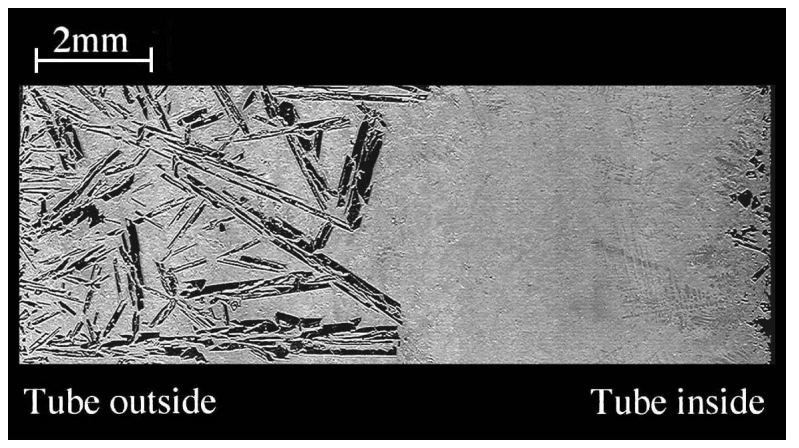

Figure 5. Micrographs showing the typical morphology of Al-Si FGM.

$$
V_{f}=100\left(\rho_{\mathrm{Al}}-\rho\right) /\left(\rho_{\mathrm{Al}}-\rho_{\mathrm{Si}}\right),
$$

where $\rho_{\mathrm{Al}}, \rho_{\mathrm{Si}}$, and $\rho$ are the density of aluminum $(=2.700$ $\left.\mathrm{Mg} / \mathrm{m}^{3}\right)$, the density of silicon $\left(=2.340 \mathrm{Mg} / \mathrm{m}^{3}\right)$, and the density measured using a densitometer with small pieces cut from specific positions parallel to the outer surface, respectively. The fraction of silicon phase in the present Al-Si FGM tube varied from approximately 60 mass $\%$ at the outer region to approximately 15 mass $\%$ at the inner region. The outer region of the tube was lighter than inner region after solidified.

At first sight, this feature contradicted our expectation that
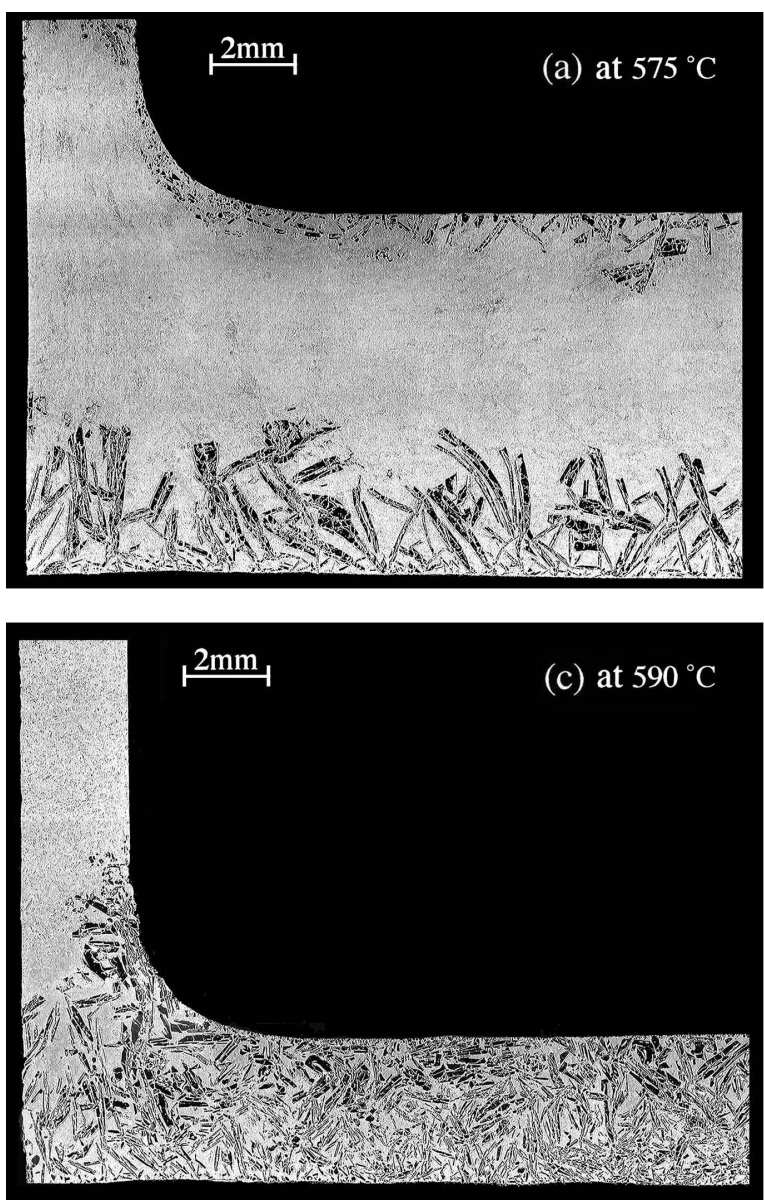

silicon would concentrate at the inner surface of the tube because the density of silicon is approximately $87 \%$ of the aluminum matrix. The density of molten Al-Si alloy is higher in the case of greater silicon content, and the density of molten Al-25 mass\% Si alloy at $1073 \mathrm{~K}\left(800{ }^{\circ} \mathrm{C}\right)$ is approximately $2.42 \mathrm{Mg} / \mathrm{m}^{3}$, as extrapolated from the values reported for $\mathrm{Si}$ alloy with less than 12 mass\% $\mathrm{Si}$ [12]. However, the density of molten aluminum at $1073 \mathrm{~K}(800$ ${ }^{\circ} \mathrm{C}$ ) is $2.33 \mathrm{Mg} / \mathrm{m}^{3}$. Thus, a molten silicon density of 2.69 $\mathrm{Mg} / \mathrm{m}^{3}$ is easily estimated using the rule of mixture. This density corresponds to the reported values for the supercooled state of silicon liquid, which range from 2.5 to $2.6 \mathrm{Mg} / \mathrm{m}^{3}[13,14]$. Therefore, the silicon constituent in the molten Al-Si alloy was gathered toward the outside of the rotating mold under centrifugal force and then silicon particles initially nucleated and subsequently grew toward the inside of the tube in the early stage of production of the FGM thick-walled tube. However, some silicon particles were finally nucleated and grew from the remaining eutectic Al-Si melt inside of the tube. Thus silicon particles were observed both inside and outside of the tube cross-section as shown in Figure 5.

\subsection{Structure of the Product Backward Extruding Using a Hydraulic Press}
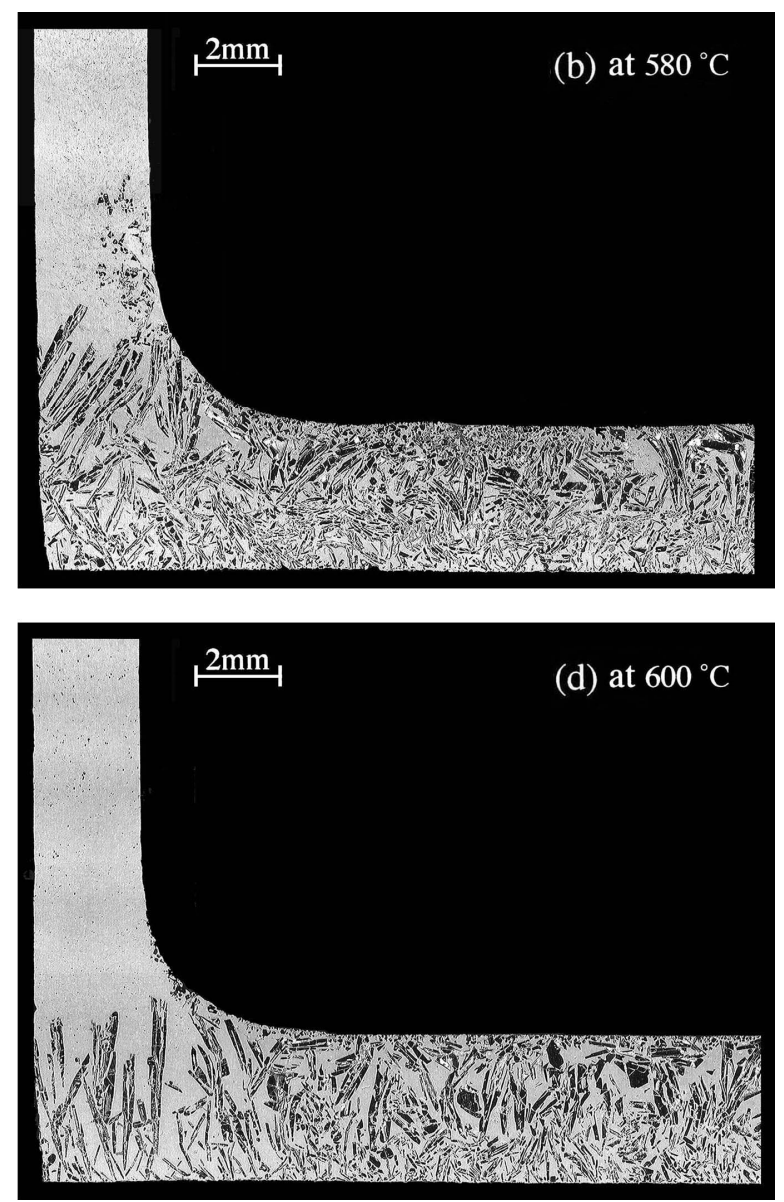

Figure 6. Micrographs showing the morphology in the cross-section of a cup extruded using a hydraulic press. 
Near-net shape cups were produced from a semi-solid backward extruding process using a hydraulic press. The morphology and distribution of silicon particles in the formed cups were also examined. Typical micrographs of the cross-sections of the cups are shown in Figure 6. The difference in thickness was attributed to the uncertainty involved in the sectioning, embedding, grinding, and polishing of the FGM cups. As shown in Figure 6 (a), only the ductile eutectic phase was slightly deformed. We subsequently confirmed that the semi-solid condition was not achieved at $848 \mathrm{~K}\left(575{ }^{\circ} \mathrm{C}\right)$, which was just below the melting point of the eutectic; thus, extruding could not be performed because of the lack of deformability. The results were quite distinctive when the tests were conducted at other higher temperatures of $853 \mathrm{~K}\left(580^{\circ} \mathrm{C}\right), 863 \mathrm{~K}\left(590^{\circ} \mathrm{C}\right)$, and $873 \mathrm{~K}\left(600^{\circ} \mathrm{C}\right)$. We confirmed that, at these temperatures, the semi-melt hypereutectic Al-Si alloy coexisted with the solid $\beta$-silicon phase ( $c f$. Figures 6 (b) to (d)). The fraction of silicon phase at the cup bottom region was condensed to greater than 70 mass $\% \mathrm{Si}$, and that at the cup wall region was less than 15 mass\% Si which was almost equal to the eutectic composition. The features corresponded qualitatively to the numerical analysis results obtained using the smoothed-particle hydrodynamics (SPH) method [15]. Thus the Al-Si FGM cups were produced successfully under a semi-solid condition.

Finer silicon particles were observed when backward extruding was performed at $853 \mathrm{~K}\left(580{ }^{\circ} \mathrm{C}\right)$ and $863 \mathrm{~K}(590$ ${ }^{\circ} \mathrm{C}$ ), compared to the distributed silicon particles in the cases of the mother FGM and other test temperatures. The results showed that most solid silicon particles beneath the punch were crushed along the compression axis and did not move toward the radial direction. However, in the case of the billet formed at $873 \mathrm{~K}\left(600{ }^{\circ} \mathrm{C}\right)$ (Figure 6(d)), most of the silicon particles exhibited features similar to those of the silicon particles in the billet, with exception of the particles beneath the inner and outer surfaces of the formed cup bottom region. In particular, the shapes of the silicon particles at the bottom left side of Figure 6(d), where the silicon particles were not directly compressed by the punch, were almost unchanged. The features indicated that the liquid portion of the semi-melt at a temperature $873 \mathrm{~K}\left(600^{\circ} \mathrm{C}\right)$ was freely passing through the gap between the solid silicon particles. Thus, a forming temperature of $873 \mathrm{~K}\left(600{ }^{\circ} \mathrm{C}\right)$ was too high to induce the preferred semi-solid forming condition and the optimum temperature appeared to be in the range from $853 \mathrm{~K}\left(580{ }^{\circ} \mathrm{C}\right)$ to $863 \mathrm{~K}\left(590{ }^{\circ} \mathrm{C}\right)$ because the fine silicon structure was achieved in this range. This tendency is consistent with the results of a refinement of intermetallics in the $\mathrm{Al}-\mathrm{Al}_{3} \mathrm{Ni}$ and $\mathrm{Al}-\mathrm{Al}_{3} \mathrm{Fe}$ FGMs where the optimum forming temperature was just above the melting point [7,8]. This observation demonstrates that shear and abrasive forces arose from the visco-plastic flow of the eutectic semi-melt during the compression stroke and refined the coarse silicon particles. Moreover, the coarse silicon particles were directly crushed into fine ones upon compression.

\subsection{Structure of Al-Si FGM Backward Extruding by a Screw Driven Tension-Compression Machine}

Similar backward extruding was performed using a screw driven tension-compression machine to examine the effect of constant compression rates on the refinement of coarse silicon particles. The tested conditions were combination of crosshead speeds of 1, 100, and $500 \mathrm{~mm} / \mathrm{min}$ and temperature of $853 \mathrm{~K}\left(580{ }^{\circ} \mathrm{C}\right)$ and $863 \mathrm{~K}\left(590{ }^{\circ} \mathrm{C}\right)$. The temperatures of $853 \mathrm{~K}\left(580{ }^{\circ} \mathrm{C}\right)$ and $863 \mathrm{~K}\left(590{ }^{\circ} \mathrm{C}\right)$ were selected on the basis of the results discussed in the previous section. A typical cross-section of an extruded Al-Si FGM cup formed at $853 \mathrm{~K}\left(580{ }^{\circ} \mathrm{C}\right)$ and at a crosshead speed of $500 \mathrm{~mm} / \mathrm{min}$ is shown in Figure 7. The features of the cross-section differed only slightly from those of the FGM cup shown in Figure 6, which was extruded using a hydraulic press. Other results are not shown here because of the similar tendencies.

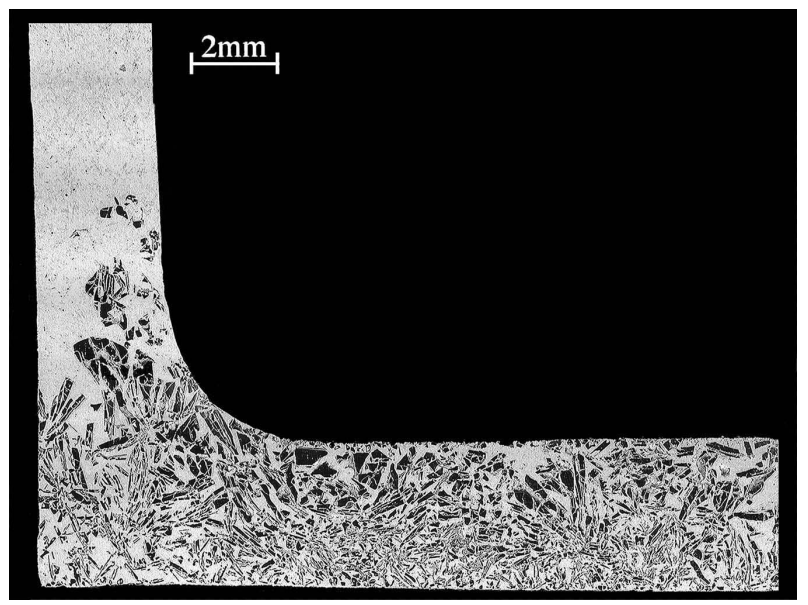

Figure 7. Micrographs showing the morphology in the cross-section of a cup extruded under conditions of $853 \mathrm{~K}\left(580^{\circ} \mathrm{C}\right)$ and $500 \mathrm{~mm} / \mathrm{min}$ using a screw-driven universal tension-compression machine.

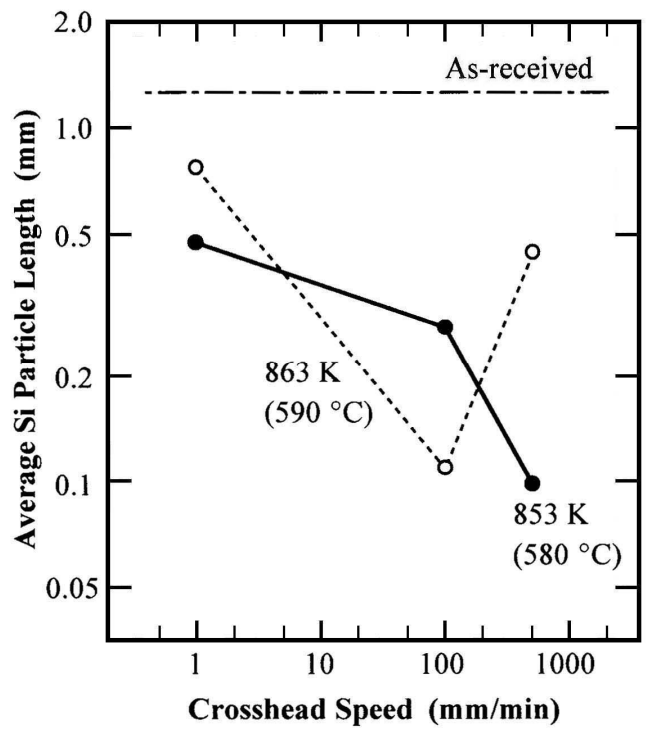

Figure 8. Variation of the average silicon particle length as a function of the compression rate. 
Figure 8 shows the dependence of silicon particle refinement on the compression rate using a double-logarithmic relationship between the average silicon particle length and the crosshead speed. The average length of the original silicon particles was $1.25 \mathrm{~mm}$, as indicated by the single dotted line in the figure. The average silicon particle lengths of the FGM cup extruded at $853 \mathrm{~K}\left(580{ }^{\circ} \mathrm{C}\right)$, which are indicated by the solid line were $0.47,0.27$, and $0.10 \mathrm{~mm}$ at crosshead speeds of 1,100 , and $500 \mathrm{~mm} / \mathrm{min}$, respectively. These results demonstrate that the refinement of silicon particles was monotonically linked to the increase of the compression rate. In contrast, the dependence of the silicon particle refinement on the compression rate in the case of the FGM cup extruded at $863 \mathrm{~K}\left(590{ }^{\circ} \mathrm{C}\right)$ differed from that of the FGM cup extruded at $863 \mathrm{~K}\left(590{ }^{\circ} \mathrm{C}\right)$. The average particle lengths of the FGM cup extrude at $863 \mathrm{~K}$ $\left(590^{\circ} \mathrm{C}\right)$, as indicated by dotted lines, were $0.75,0.11$, and $0.46 \mathrm{~mm}$ at crosshead speeds of 1,100 , and $500 \mathrm{~mm} / \mathrm{min}$, respectively, and the distribution pattern showed a concave curve. These results indicate that finer silicon particles were not always accompanied by a faster strain rate and that the dependence appeared to be complicated. The optimum semi-solid forming conditions may not depend only on maintaining a temperature just above the eutectic melting point but may also depend on the interactions between the working temperature and the working rate.

\subsection{Estimation of the Requirement for Refinement of Silicon Particles}

The determination of the optimum conditions for the refinement of silicon particles via semi-solid forming in an Al-Si FGM requires a comprehensive analysis of complicated deformation resistance that accompanies the change of the material state from solid, semi-solid, to melt. One factor to be considered in the discussion of the experimental results shown in Figure 8, which indicate that the working rate, i.e., the strain rate and shear rate, was not the predominant factor governing the silicon particle refinement at a specific temperature, is the reverse tendency for a higher working rate to increase the plastic deformation resistance [16] and decrease the viscous flow resistance [17]. Moreover, both the plastic and viscous flow resistances decreased depending on the increase in the working temperature. Therefore, in visco-plastic forming applied to the mixture of solids and liquids, the effect of the viscosity on the refinement of coarse silicon particles - in which metallic components transformed from solids, semi-solids, and then liquids, accompanied by an increase in the working temperature - must be considered.

The strain rate increases as the compression rate increases and then the shear rate increases. The increase in shear rate accompanies a decrease in the viscosity [17]. For the evaluation of a process concerning with the silicon particle refinement, Figure 9 showed the relationship between the viscosity and the silicon particle size on a double-logarithmic chart. The filled and open circles represented the corresponding values at working temperatures of $853 \mathrm{~K}$ (580 $\left.{ }^{\circ} \mathrm{C}\right)$ and $863 \mathrm{~K}\left(590{ }^{\circ} \mathrm{C}\right)$, respectively, and the numbers represent the applied working crosshead speed $[\mathrm{mm} / \mathrm{min}]$, i.e., the compression rate. The viscosities of each test condition were obviously unknown; graduated scale along the abscissa was not shown in Figure 9. However, the logarithmic scales of the estimated viscosity shown by gray dotted lines were proportional to the compression rate for each test temperature. The relative positions were approximated on the basis of the concept that higher crosshead speed decreased the viscosity and higher working temperature also decreased the viscosity and resulted in wider variation. Then we draw an approximate broken line that indicated the idealized variation of silicon particle size as a function of the qualitatively estimated viscosity. The transition point of the dominant factor governing the flow process from a plastic flow to a viscous flow appeared to occur in the range of approximately from $500 \mathrm{~mm} / \mathrm{min}$ at $853 \mathrm{~K}\left(580{ }^{\circ} \mathrm{C}\right)$ to $100 \mathrm{~mm} / \mathrm{min}$ at 863 $\mathrm{K}\left(590^{\circ} \mathrm{C}\right)$ from this estimation. The viscous flow becomes a dominant influence on the deformation and then the effect on particle refining diminished at temperatures over $873 \mathrm{~K}(600$ ${ }^{\circ} \mathrm{C}$ ) because the visco-plastic flow resistance decreased rapidly with increasing temperature, as suggested by the results in Figure 6 (d). We inferred that the transition of the flow-process dominant factor caused the seemingly contradictory results of finer silicon particles not always accompanying a faster working rate as shown in Figure 8.

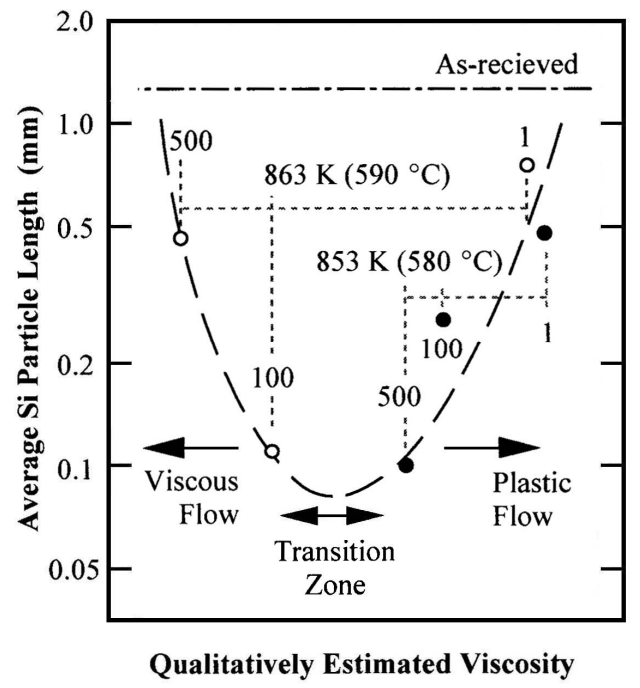

Figure 9. Schematic of the average silicon particle length as a function of the qualitative estimated viscosity. Gray dotted lines show the estimated scale for each test temperatures and numbers indicate the crosshead speed [mm/min].

To expand the possible application of hypereutectic $\mathrm{Al}-\mathrm{Si}$ alloy, we examined the optimum semi-solid forming condition of Al-Si FGM in relation to the silicon particle refinement based on the experimental results. Although higher shear rate operation produced a fine structure at approximately $853 \mathrm{~K}\left(580{ }^{\circ} \mathrm{C}\right)$ and/or up to $863 \mathrm{~K}\left(590{ }^{\circ} \mathrm{C}\right)$, a faster visco-plastic flow was not effective for refinement coarse silicon particles at temperatures over $873 \mathrm{~K}\left(600^{\circ} \mathrm{C}\right)$ because the visco-plastic flow resistance decreased and the lower-viscosity melt easily passed through the gaps between 
the silicon particles. The preferred condition conflicted with the plastic flow resistance and the viscous flow resistance under not too soft, not too hard. To achieve refinement of silicon particles, we can reasonably conclude that high-speed operation at approximately $853 \mathrm{~K}\left(580{ }^{\circ} \mathrm{C}\right)$ should be the optimum operating conditions. It closely agrees with a series of reports that the semi-solid forming just above the melting point is preferable for preparing finer intermetallics in aluminum-based FGMs [7,8]. Our results indicated that the use of a semi-solid forming process for the fabrication of FGM products from hypereutectic Al-Si alloys should be feasible.

\section{Summary}

In a series of studies on FGMs, we conducted experiments toward the practical usage of Al-Si FGM by analyzing the semi-solid forming products of Al-Si FGM cups produced via backward extruding and comparing the results with those obtained for a master Al-Si FGM tube manufactured via a vacuum centrifugal method using hypereutectic Al-25 mass $\% \mathrm{Si}$ alloy. We drew the following conclusions on the basis of this work:

1) Contrary to our expectations, the fraction of silicon phase varied from greater than 60 mass $\%$ at the outer surface to 15 mass $\%$ at the inner surface of the FGM thick-walled tube manufactured by the centrifugal method because of the greater density of molten silicon compared to that of the eutectic melt.

2) Al-Si FGM cups were successfully formed at $853 \mathrm{~K}$ $\left(580^{\circ} \mathrm{C}\right), 863 \mathrm{~K}\left(590{ }^{\circ} \mathrm{C}\right)$, and $873 \mathrm{~K}\left(600{ }^{\circ} \mathrm{C}\right)$ by a semi-solid forming via a backward extruding using a 200-kN hydraulic press. The optimum operating temperatures were approximately $853 \mathrm{~K}\left(580^{\circ} \mathrm{C}\right)$ and $863 \mathrm{~K}\left(590^{\circ} \mathrm{C}\right)$ because the preferred fine silicon structure was obtained at these temperatures but not observed at $873 \mathrm{~K}\left(600^{\circ} \mathrm{C}\right)$.

3) Under a constant compression rate test at $853 \mathrm{~K}(580$ ${ }^{\circ} \mathrm{C}$ ) and $863 \mathrm{~K}\left(590^{\circ} \mathrm{C}\right)$ using a $100-\mathrm{kN}$ screw driven tension-compression machine, a faster compression rate did not always result in the finer silicon particle and the dependence appeared to be complicated.

4) Both viscous flow and plastic flow act by competing for achieving refinement of silicon particles and operation under moderate softening and/or melting of the alloy will expand the possibility of using Al-Si FGM as a practical elemental material.

\section{Acknowledgements}

This research work has been partially support by the Light Metal Educational Foundation Inc. of Japan.

\section{References}

[1] B.K. Prasad, K. Venkateswarlu, O.P. Modi, A.K. Jha, S. Das, R.
Dasgupta, and A.H. Yegneswaran, "Sliding Wear Behavior of Some Al-Si Alloys: Role of Shape and Size of Si Particles and Test Conditions", Metallurgical and Materials Transactions A, Vol. 29A, No. 11 (1998), pp. 2747-2752.

[2] S. Uemura, Y. Noda, Y. Shinohara, and Y. Watanabe, Development and Application of Functionally Graded Materials (2003), CMC Publishing Co. Ltd.

[3] Y. Fukui, "Fundamental Investigation of Functionally Gradient Material Manufacturing System using Centrifugal Force", JSME International Journal Series III, Vol. 34, No. 1 (1991), pp. 144-148.

[4] Y. Fukui, N. Yamanaka, and Y. Enokida, "Bending strength of an Al-Al3Ni functionally graded material", Composites B, Vol. 28B, Nos. 1,2 (1997), pp. 37-43.

[5] Y. Watanabe, N. Yamanaka, and Y. Fukui, "Control of composition gradient in a metal-ceramic functionally graded material manufactured by the centrifugal method", Composites A, Vol. 29A, Nos. 5,6 (1998), pp. 595-601.

[6] Y. Watanabe, N. Yamanaka, and Y. Fukui, "Wear behavior of Al-Al3 Ti Composite Manufactured by a Centrifugal Method", Metallurgical and Materials Transactions A, Vol. 30A, No. 11 (1999), pp.3253-3261.

[7] Y. Fukui, H. Okada, N. Kumazawa, and Y. Watanabe, "Near Net Shape Forming of Al-Al3Ni FGM over Eutectic Melting Temperature", Metallurgical and Materials Transactions A, Vol. 31A, No. 10 (2000), pp. 2627-2636.

[8] K. Yamagiwa, Y. Watanabe, K. Matsuda, Y. Fukui, and P. Kapranos, "Characteristic of Al-Al3Fe Eco- Functionally Graded Material through Near-Net-Shape Forming over Eutectic Melting Temperature", Materials Science and Engineering A, Vol. A416, Nos. 1-2 (2006), pp. 80-91.

[9] ASM Handbook vol.15, 9th ed., ASM INTERNATIONAL, Metals Park, (1988), pp.327-338.

[10] M.C. Flemings, "Behavior of Metal Alloys in the Semisolid State", Metallurgical Transactions A, Vol.22A, No.5 (1991), pp.957-981.

[11] T. B. Massalski, Binary Alloy Phase Diagrams, Second Edition Plus Updates on CD-ROM Version 1.0 (1996), ASM International.

[12] T. Magnusson, and L. Arnberg, "Density and Solidification Shrinkage of Hypoeutectic Aluminum-Silicon Alloys", Metallurgical and Materials Transactions A, Vol. 32A, No. 10 (2001), pp. 2605-2613.

[13] K. Ohsaka, S.K. Chung, W.K. Rhim, and J.C. Holzer, "Densities of Si determined by an image digitizing technique in combination with an electrostatic levitator", Applied Physics Letters, Vol. 70 (1997), pp. 423-425.

[14] R.K. Endo, Y. Fujihara, and M. Susa, "Calculation of density and heat capacity of silicon by molecular dynamics simulation" High Temperatures - High Pressures, Vol. 35/36 (2006), pp. 505-512.

[15] H. Takamiya, H. Okada, Y. Sakai, and Y. Fukui, "Smoothed particle hydrodynamics analysis on semi-solid metal forming process", Japan Journal of Industrial and Applied Mathematics, Vol. 28 (2011), pp. 183-203. 
[16] E.W. Hart, "Theory of the tensile test", Acta Metallurgica, Vol.15, No.2 (1967), pp. 351-355.

[17] J.A. Yurko, and M.C. Flemings, "Rheology and
Microstructure of Semi-Solid Aluminum Alloys Compressed in the Drop Forge Viscometer", Metallurgical and Materials Transactions A, Vol. 33A, No.8 (2002), pp. 2737-2746. 